\title{
An Analysis of Supersonic Flows with Low-Reynolds Number Compressible Two-Equation Turbulence Models Using LU Finite Volume Implicit Numerical Techniques
}

J. Lee

Sverdrup Technology, Inc.

Lewis Research Center Group

Brook Park, Ohio

April 1994

Prepared for

Lewis Research Center

Under Contract NAS3-25266

National Aeronautics and

Space Administration 


\title{
An Analysis of Supersonic Flows with Low-Reynolds Number Compressible Two-Equation Turbulence Models Using LU Finite Volume Implicit Numerical Techniques
}

\author{
J. Lee \\ Sverdrup Technology, Inc. \\ Lewis Research Center Group \\ Brook Park, Ohio 44142
}

\begin{abstract}
A generalized flow solver using an implicit Lower-Upper(LU) diagonal decomposition based numerical technique has been coupled with three low-Reynolds number $k-\varepsilon$ models for analysis of problems with engineering applications. The feasibility of using the LU technique to obtain efficient solutions to supersonic problems using the $\mathrm{k}-\varepsilon$ model has been demonstrated. The flow solver is then used to explore limitations and convergence characteristics of several popular two equation turbulence models. Several changes to the LU solver have been made to improve the efficiency of turbulent flow predictions. In general, the low-Reynolds number $k-\varepsilon$ models are easier to implement than the models with wall-functions, but require much finer nearwall grid to accurately resolve the physics. The three $k-\varepsilon$ models use different approaches to characterize the near wall regions of the flow. Therefore, the limitations imposed by the near-wall characteristics have been carefully resolved. The convergence characteristics of a particular model using a given numerical technique are also an important, but most often overlooked, aspect of turbulence model predictions. It is found that some convergence characteristics could be sacrificed for more accurate near-wall prediction. However, even this gain in accuracy is not sufficient to model the effects of an external pressure gradient imposed by a shockwave/boundary-layer interaction. Additional work on turbulence models, especially for compressibility, is required since the solutions obtained with base line turbulence are in only reasonable agreement with the experimental data for the viscous interaction problems.
\end{abstract}

\section{Nomenclature}

\begin{tabular}{|c|c|c|c|}
\hline $\mathrm{A}, \mathrm{B}$ & Convective Jacobians & M & Mach Number, $\left(=\mathrm{u} /(\bar{\gamma} R T)^{1 / 2}\right)$ \\
\hline $\mathrm{a}, \mathrm{b}, \overline{\mathrm{g}}, \overline{\mathrm{h}}$ & $\begin{array}{l}\text { Constants in Van-Driest Transformation, } \\
\text { Eqn } 19 \text {, Eqn } 20\end{array}$ & $\mathrm{~N}$ & $\begin{array}{l}\text { Conservative/Non-conservative Transfor- } \\
\text { mation Vector. }\end{array}$ \\
\hline $\mathrm{D}$ & Difference Operator & $P_{k}$ & Turbulence Production \\
\hline $\mathrm{C}_{\varepsilon 1} \mathrm{C}_{\varepsilon 2}$ & Turbulence Model Constants, Table 1 & $\operatorname{Pr}$ & Prandtl Number \\
\hline $\mathrm{C}_{\mu}$ & Eddy Viscosity Constant, $(=0.09)$ & $\mathrm{P}, \mathrm{p}$ & Pressure \\
\hline $\mathrm{C}_{\mathrm{f}}$ & Skin Friction Coefficient & $\mathrm{R}_{\mathrm{t}}$ & Turbulent Reynolds Number $\left(=\mathrm{k}^{2} / v \varepsilon\right)$ \\
\hline $\mathrm{C}_{\mathrm{t}}$ & A Parameter in Eqn. 20 & $\mathrm{R}_{\mathrm{k}}$ & Near Wall Turbulence Reynolds Number \\
\hline $\mathrm{E}, \mathrm{F}, \mathrm{Q}$ & Conservative Vectors & & $\left(=k^{1 / 2} y_{n} / v\right)$ \\
\hline $\mathrm{f}_{\mu}$ & Wall Damping Function, Eqns 4, 5, 6 & $\mathrm{R}$ & Gas Constant \\
\hline$H$ & Source Vector & $\overline{\mathrm{r}}$ & Recovery factor $\left(=\operatorname{Pr}^{1 / 3}\right)$ \\
\hline $\mathrm{H}$ & Enthalpy & $\mathrm{T}$ & Temperature \\
\hline $\mathrm{h}$ & Cell Volume & $\mathrm{T}_{\mathrm{u}}$ & Turbulence Intensity \\
\hline I & Identity Vector & $\mathrm{u}, \mathrm{v}$ & Mean Velocity Constants \\
\hline $\mathrm{k}$ & Turbulent Kinetic Energy & $\mathrm{U}_{\tau}$ & Friction Velocity $\left(=\left(\tau_{\mathrm{w}} / \rho\right)^{1 / 2}\right)$ \\
\hline l & Length Scale, Eqn 22 & $\mathrm{x}, \mathrm{y}$ & Coordinates \\
\hline $\mathrm{M}_{\eta}, \mathrm{M}_{\xi}$ & Implicit Viscous Terms & $\mathrm{y}^{+}$ & Non-Dimensional Distance \\
\hline
\end{tabular}




\begin{tabular}{|c|c|}
\hline \multicolumn{2}{|c|}{ Symbols } \\
\hline$\varepsilon$ & Dissipation \\
\hline$\delta$ & Boundary Layer Thickness \\
\hline & Maximum spectral radii $(=\operatorname{MAX}(\lambda))$ \\
\hline $\bar{\gamma}$ & Ideal gas constant $(=1.4)$ \\
\hline$\lambda$ & Local spectral radii \\
\hline & Low Reynolds number terms \\
\hline & Viscosity \\
\hline$d_{t}$ & Eddy Viscosity $\left(=\mathrm{C}_{\mu} \mathrm{f}_{\mu} \mathrm{k}^{2} / \varepsilon\right)$ \\
\hline & Kinematic Viscosity \\
\hline$\eta, \xi$ & Body fitted coordinate system \\
\hline & Wake Constant $(=0.50)$ \\
\hline & Viscous diffusion \\
\hline 5 & Viscous Diffusion Coefficient, Table 1 \\
\hline & Shear Stress \\
\hline \multicolumn{2}{|c|}{ Subscripts and superscripts } \\
\hline 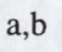 & With respect to the Directions \\
\hline & Boundary Condition \\
\hline & With respect to Turbulent Kinetic Energy \\
\hline & pseudo time index \\
\hline & With respect to Turbulence \\
\hline & With respect to Viscous Terms \\
\hline & Transformed Variable \\
\hline & Freestream \\
\hline & At the inlet \\
\hline
\end{tabular}

During the past several years, significant progress has been made in the development of new turbulence models and in the understanding of the turbulence model limitations. A majority of modelling efforts have been directed towards three classes of turbulence models with various degrees of physical complexity. It was previously shown that the simplest turbulence models can be used to perform reasonably accurate combustor flowfield predictions. ${ }^{1}$ This work also showed that a more accurate turbulence model may be needed to resolve the desired performance characteristics of the combustors. Therefore, prior to studying a very complex problem in a combustor flowfield, the turbulence model and the numerical techniques in non-reacting situations were studied and are described herein.

The simplest of the turbulence models usually assume length scale behaviors and are known as the zero equation algebraic eddy viscosity models. These models utilize local equilibrium turbulent scale distributions and the mean shear information to complete the Boussinseq hypotheses necessary to close the averaged Navier-Stokes equations. The models in this class are widely used in Computational
Fluid Dynamic(CFD) computations because of their simplicity. However, the major Achilles heel of these models is the limitation imposed by the equilibrium assumption used in the development of the length scale profiles. In flowfields involving non-equilibrium phenomena, such as separation, sudden removal or addition of pressure gradient, injection, suction, wall temperature gradient and roughness, the predictions made using these models tend to be poor because the required model physics often exceed the equilibrium assumption. ${ }^{2}$

The second class of model retains the eddy viscosity/ Boussinseq approximations. However, these models use transport equations to find the required turbulent length and velocity scales of the flowfield to complete the eddy viscosity formulation and therefore these models are not limited to equilibrium situations. Unfortunately, a majority of the modeling parameters and the near-wall formulations are optimized only for simple flow situations. Therefore, the limitations of these models must be carefully explored in complex flow situations. There are many viable suggestions as to which quantities should be used to characterize the flowfield. The turbulent kinetic energy is the first dependent variable used in many of these models. However, the choice of the second dependent variable required to determine the length scales varies. Some of the more successful choices are dissipation rate $(\varepsilon),{ }^{3}$ specific dissipation rate $(\omega),{ }^{4}$ and dissipative time scale $(\tau){ }^{5}$

For the third class of turbulence model, the Reynolds $\operatorname{stresses}\left(\tau_{\mathrm{ij}}\right)$ are used directly to close the mass(Favre)-averaged equations. This is usually called the second order closure model or the Reynolds stress model. The second order closure model formulation eliminates the isotropic assumption in which the eddy viscosity is aligned only with the mean shear. However, this gain in generality does not always translate directly into improved solution accuracy. ${ }^{6}$ Recently, several investigators have reported that complex flow situations can indeed be resolved using the Reynolds Stress model and very promising predictions have been reported by Launder-Shima ${ }^{7}$ and Lee et al. ${ }^{8}$ However, these works have also shown that the solution to full Reynolds-stress models can be achieved only through considerable computational effort which is undesirable in engineering analysis.

The most wisely used and extensively developed of the two equation eddy viscosity models is the $\mathrm{k}-\varepsilon$ model. In this formulation the length scale and the velocity scale needed to determine the eddy viscosity are computed from the modeled turbulent kinetic energy and dissipation rate transport equations. The $\mathrm{k}-\varepsilon$ model has been used in a wide variety of low Mach number problems. There are, however, several well known $k-\varepsilon$ model deficiencies. A major deficiency of the model is its inability to handle no-slip surfaces. There are two techniques commonly used to resolve this deficiency. Extensive review of this deficiency 
and analysis of the various model correction formulations have been conducted by Rodi ${ }^{9}$, Coakley and Huang, ${ }^{10}$ and subsequently by Michelassi and Shih. ${ }^{11}$ The first method replaces the turbulence transport equations in the near-wall regions with a known solution, usually based on the loglaw assumption. This is usually known as the high-Reynolds number/wall-function approach. The second method is to modify the transport equations so that the singular behavior in the near wall region is removed and much simpler zero boundary conditions are satisfied. Only the lowReynolds number models were studied within this work.

A majority of the models previously discussed were developed for incompressible flows and have not been extensively tested for high Mach number flows. The compressible versions of these models, applicable in high Mach number flows, are just being introduced and potential problems are being realized. ${ }^{10}$ The early compressible turbulence models have been shown to be inadequate due to the lack of dilatational effects in the original formulations. Therefore, extensive efforts ${ }^{7,8}$ are being made to construct a physically optimal turbulence model for high Mach number compressible flows. These efforts have resulted in considerable advances being made in the development of models for various dilatation terms, such as dilatation pressure and dilatation dissipation. ${ }^{12,13,14}$ However, these new modeling concepts have not yet been extensively tested for combustor flowfields. Therefore, in this work we have coupled the latest two-dimensional implicit finite volume numerical technique with the latest compressible two equation turbulence models, including the effects of dilatational terms, to develop a generalized CFD solver that can be economically used to solve a variety of complex internal flow problems of engineering interest.

The convergence characteristic is an important, but most often overlooked, aspect of any analysis using turbulence models. ${ }^{10}$ In many instances, the numerical techniques will introduce unwanted transients, usually associated with numerical error, that can destabilize the numerics. This problem becomes more acute when convergence acceleration techniques are used. In some situations, local changes can translate into irreversible and non-physical turbulence characteristics. A typical example of this situation occurs in the computation of the freestream region, where the turbulence problem should reduce to a natural decay, but more often the computed turbulent energy decays more rapidly than expected because of the numerical dissipation. This unexpected dissipation rate can cause the turbulent kinetic energy to decay to non-physical values, causing numerical instabilities to occur and consequently leading to convergence failure. There are a number of different suggestions for removing this limitation. The first is to limit the turbulence production during the iteration process to physically realistic levels. A second method is to start the solution process from a previously converged solution. Neither of these methods is very palatable in engineering applications. Recently, Huang and Coakley ${ }^{15}$ demonstrated that neither of these arrangements is necessary if the numerics has certain Total Variation Diminishing(TVD) properties and if the impending oscillations are not allowed to turn negative. However, it is difficult to maintain TVD-like properties for all of the existing numerical schemes. Therefore, we have attempted to incorporate some of the proposed numerical behavior into the solvers.

The focus of this work is to describe the development of an efficient LU solver for the low-Reynolds number $k-\varepsilon$ models and to address some of the key issues regarding the turbulence model physics and their implications in high speed internal flow predictions.

\section{Generalized Turbulence Model Formulations}

The governing equations of a typical two equation model can be written in the conservative form:

$$
\frac{\partial Q}{\partial t}+\frac{\partial}{\partial x}\left(F-F_{v}\right)+\frac{\partial}{\partial y}\left(G-G_{v}\right)=H
$$

where

$$
\begin{gathered}
Q=\left[\begin{array}{c}
\rho k \\
\rho \varepsilon
\end{array}\right] \\
F-F_{v}=\left[\begin{array}{c}
\rho k u-\sigma_{k x} \\
\rho \varepsilon u-\sigma_{\varepsilon x}
\end{array}\right] \quad G-G_{v}=\left[\begin{array}{l}
\rho k v-\sigma_{k y} \\
\rho \varepsilon v-\sigma_{\varepsilon y}
\end{array}\right] \\
H=\left[\begin{array}{c}
P_{k}-\rho \varepsilon \\
C_{\varepsilon 1} \frac{\varepsilon}{k} P_{k}-C_{\varepsilon 2} f_{\varepsilon} \frac{\rho \varepsilon^{2}}{k}
\end{array}\right]+\Lambda
\end{gathered}
$$

and

$$
\sigma_{k x}=\left(\mu+\frac{\mu_{t}}{\bar{\sigma}_{k}}\right) \frac{\partial k}{\partial x} \quad \sigma_{\varepsilon x}=\left(\mu+\frac{\mu_{t}}{\bar{\sigma}_{\varepsilon}}\right) \frac{\partial \varepsilon}{\partial x}
$$

The form of $\sigma_{\mathrm{ky}}$ and $\sigma_{\varepsilon y}$ are similar to above. The production, shear stress $\left(\tau_{x y}\right)$, and normal stress $\left(\tau_{x x}, \tau_{y y}\right)$ terms are defined as follows,

$$
P_{k}=\rho \tau_{x y}\left[\frac{\partial u}{\partial y}+\frac{\partial v}{\partial x}\right]+\rho \tau_{x x} \frac{\partial u}{\partial x}+\rho \tau_{y y} \frac{\partial v}{\partial y}
$$




$$
\begin{gathered}
\rho \tau_{y y}=\mu_{t}\left[2 \frac{\partial v}{\partial y}-\frac{2}{3}\left[\frac{\partial u}{\partial x}+\frac{\partial v}{\partial y}\right]\right]-\frac{2}{3} \bar{\rho} k \\
\rho \tau_{x y}=\mu_{t}\left[\frac{\partial u}{\partial y}+\frac{\partial v}{\partial x}\right] \\
\rho \tau_{x x}=\mu_{t}\left[2 \frac{\partial u}{\partial x}-\frac{2}{3}\left[\frac{\partial u}{\partial x}+\frac{\partial v}{\partial y}\right]\right]-\frac{2}{3} \bar{\rho} k
\end{gathered}
$$

where the turbulence modeling constants vary from model to model and are shown in Table 1. All of the quantities appearing above are mass $\left(\right.$ Favre $\left.^{16}\right)$ averaged, i.e. $u=$ $\rho u / \bar{\rho}$, and $\mathrm{k}=\bar{\rho} k / \bar{\rho}$. The density $(\rho)$ is conventionally averaged.

In general, low-Reynolds number $k-\varepsilon$ models use modified transport equations, where a fictitious dissipation $(\bar{\varepsilon})$ rather than the real dissipation $(\varepsilon)$ is used. The fictitious dissipation is usually defined as $\bar{\varepsilon}=\varepsilon-\varepsilon_{\text {wall }}$. There are typically three different types of low-Reynolds number $k-\varepsilon$ models. All three employ additional wall damping to satisfy any additional near wall physics. The first type uses the flowfield characteristics, such as curvature of the turbulent kinetic energy $\left(\varepsilon_{\text {wall }}=2 v\left(d^{2} k / d y^{2}\right)^{2}\right)$, to satisfy the near wall asymptotic behavior, while at same time avoiding the singularity caused by the no-slip boundary condition. The original $k-\varepsilon$ model formulation proposed by Launder and Sharma ${ }^{17}$ is typical of this type of formulation. The second type of low-Reynolds number $\mathrm{k}-\varepsilon$ model, proposed by Chien, ${ }^{18}$ uses the wall normal distance $\left(\varepsilon_{\text {wall }}=2 v \mathrm{k} / \mathrm{y}_{\mathrm{n}}{ }^{2}\right)$ to develop the necessary near wall characteristics. The third type of low-Reynolds number formulation makes no attempt to correct the transport equation; rather, finite nearwall characteristics are used as boundary conditions. The low Reynolds number model proposed by Shih ${ }^{19}$ is typical of the models that avoid the zero boundary condition and therefore the singularity. In practice, the low-Reynolds number $k-\varepsilon$ models are easier to numerically implement than a high Reynolds number model. However, the lowReynolds number models also require much higher near wall grid resolution. Typical modeling constants of these $\mathrm{k}$ $\varepsilon$ models are shown in Table 1 .

\begin{tabular}{|l|c|c|c|c|c|}
\hline Models & $C_{\varepsilon 1}$ & $C_{\varepsilon 2}$ & $C_{\mu}$ & $\bar{\sigma}_{\mathrm{k}}$ & $\bar{\sigma}_{\varepsilon}$ \\
\hline \hline Chien & 1.35 & 1.80 & 0.09 & 1.0 & 1.30 \\
\hline $\begin{array}{c}\text { Launder } \\
\text { Sharma }\end{array}$ & 1.45 & 1.92 & 0.09 & 1.0 & 1.30 \\
\hline Shih & 1.45 & 2.00 & 0.09 & 1.0 & 1.30 \\
\hline
\end{tabular}

Table 1: Turbulence Model Parameters
I.a Low-Reynolds Number k-e model Formulation of Launder and Sharma(1976)

As previously discussed, the $\mathrm{k}-\varepsilon$ models employ additional damping functions to satisfy the near wall physics. All of the models try to satisfy $\mathrm{y}_{\mathrm{n}}{ }^{2}$ behavior of the turbulent kinetic energy using slightly different damping functions along with the $\varepsilon_{\text {wall }}$ formulation. The LaunderSharma model employs a near wall damping formulation that satisfies the invariance principle by using only near wall scalar characteristics. Therefore, the damping functions are independent of the grid coordinate system. Thus, for complex wall geometries additional modeling assumptions are not needed and the near-wall characteristics are uniquely defined. Unfortunately, this model is more sensitive to numerical error than Chien's model. Similar difficulties have been observed by Gerolymos. ${ }^{18}$ The reason for these difficulties lies in the sub-layer region of a typical boundary-layer computation where the initial transient causes a large and unphysical production of turbulent energies which sometimes cannot be readjusted to the field. This difficulty was controlled using a limiter on the dissipations in the work of Gerolymos. ${ }^{20}$ However, we have found that such a limiter is not needed if a low CFL number is used to reduce the initial transients. The low-Reynolds number correction terms for this model are defined as follows,

$$
\begin{gathered}
\Lambda=\left[\begin{array}{c}
-2 \mu(\nabla \sqrt{k})^{2} \\
\frac{2}{\rho} \mu \mu_{t}\left(\nabla^{2} U\right)^{2}
\end{array}\right] \\
f_{\mu}=\exp \left(\frac{-3.5}{\left(50+R_{t}\right)^{2}}\right)
\end{gathered}
$$

\section{I.b Low Reynolds Number k-e model Formulation of Chien(1976)}

A low-Reynolds number $\mathrm{k}-\varepsilon$ model with VanDriest type near-wall damping function and algebraic near wall formulation was proposed by Chien. ${ }^{18}$ This lowReynolds number formulation is not Gallean-invariant and is not independent of the grid coordinate system used. Therefore, a major disadvantage of this formulation is that for complex geometries the near wall characteristics may not be uniquely defined; for some cases, there may be wide range of possibilities. However, for the problems investigated, this model seems to have the best overall convergence behavior and numerical stability of the three models explored. Furthermore, since the test problems investigated are bounded by a single no-slip surface, lack of invariance was not a problem. The low-Reynolds 
number correction terms for the Chien model are defined as follows:

$$
\begin{aligned}
& \Lambda=\left[\begin{array}{c}
-2 \mu \frac{k}{y_{n}^{2}} \\
-2 \mu \frac{\varepsilon}{y_{n}^{2}} \exp \left(-0.5 y_{n}^{+}\right)
\end{array}\right] \\
& f_{\mu}=1.0-\exp \left(0.0115 y_{n}^{+}\right)
\end{aligned}
$$

where $y_{n}$ is the nearest surface normal distance.

\section{I.c Low-Reynolds Number k-e model Formulation of Shih and Lumley(1990)}

This model formulation involves a hybrid approach in which the near wall characteristics are modeled as boundary conditions and not as modified terms. The typical form of the low-Reynolds number damping terms are defined as follows:

$$
\Lambda=\left[\begin{array}{c}
0.0 \\
\frac{1}{\rho} \mu \mu_{t}\left(\nabla^{2} U\right)^{2}
\end{array}\right]
$$

$$
f_{\mu}=\sqrt{\left(1+\exp \left(a_{1} R_{k}+a_{3} R_{k}+a_{5} R_{k}\right)\right)}
$$

where the damping constants $a_{1}, a_{3}$, and $a_{5}$ are defined as $-1.5 \times 10^{-4},-1.0 \times 10^{-9}$ and $-5.0 \times 10^{-10}$, respectively. Note that the dissipation term in the turbulent kinetic energy equation has not been modified. However, this formulation uses the surface normal distance in the damping function, as does Chien's formulation. Therefore, this model does not satisfy the invariant principal; further, it is sometimes difficult to determine the proper surface normal distance in complex flow situations. However, because the normal distance is used only in an exponential term of the damping function formulation, this model is far less sensitive to the choice of the surface normal distance than the Chien formulation, where the surface normal distance is used directly as a dissipation correction term.

\section{I.d Compressibility Effects}

The effect of compressibility is an important consideration in developing a high speed turbulence model. Essentially, disregarding the dilatation effects will lead to under representation of the additional dissipation caused by volumetric or density fluctuations. Therefore, the effects of both the dilatation dissipation and pressure transport terms are usually modeled using the solenoidal dissipation and the turbulent Mach number $\left(\mathrm{M}_{\mathrm{t}}\right)$ or density variance. ${ }^{14}$ The simplest form of the proven modeling concept proposed by Zeman ${ }^{12}$ and Sarkar ${ }^{13}$ has been used to close the dilatation dissipation and the pressure dilatation effects. Since nearwall turbulence is considered in this study, a more appropriate form with correct near-wall limit suggested by Wilcox ${ }^{21}$ has been implemented.

$$
\varepsilon_{d}=\alpha\left(M_{t}^{2}-0.06\right) \varepsilon H\left(M_{t}-0.25\right)
$$

where $\mathrm{H}(>1.0)=1.0$ and $\mathrm{H}(<0)=0.0$.

\section{Numerical Techniques}

Implicit LU finite volume numerical techniques were used to solve the Favre averaged Navier-Stokes equations along with the transport equations of turbulent kinetic energy and dissipation. This LU numerical technique was originally proposed by Yoon and Jameson. ${ }^{22}$ The details of the Successive Symmetric Over Relexation(SSOR) scheme are well documented. Therefore, only the specifics dealing with the numerics of the turbulent transport equations are discussed here. One advantage of the LU diagonal decomposition technique is that the large CFL number marching ability of an implicit numerical schemes to be retained without the large block matrix operations. Furthermore, when the LU decomposed implicit operators are combined with a directionally sweeping procedure, the solution requires only a series of simple back substitutions.

The approximate splitting method proposed by Jameson ${ }^{22}$ based on the maximum spectral radii has been used in this work. This leads to Lower and Upper matrices which then can be solved using scalar inversions. A more rigorous LU formulation Steger and Warming(SW) ${ }^{23}$ fluxvector splitting procedure was also studied. In general, the splitting process reduces the complexity of the numerical operations in return for reduced convergence behavior $\left(\mathrm{Hirsh}^{24}\right)$. In order to regain some of the lost convergence speed and accuracy, the viscous terms have been added implicitly and the explicit side of the numerics was refined using an upwind technique. These two improvements further enhance the skin friction convergence. Typical convergence characteristics of these numerical techniques are compared in Figure 1. The time history of the skin friction coefficient at the last wall surface station of a boundary-layer is shown in the top figure, and the total residual of the mean terms is shown in the lower figure. This figure shows that a reasonable convergence behavior can be achieved using the numerical techniques investigated. This figure also shows that the SW left-hand side and upwind differencing(UP) improve the convergence speed. Typically, approximately 500 to 1000 
iterations based on the third significant figure of the skin friction predicted separate the three options shown in this figure. This figure shows that the SSOR technique with central differencing $(C D)$ has the slowest convergence rate.

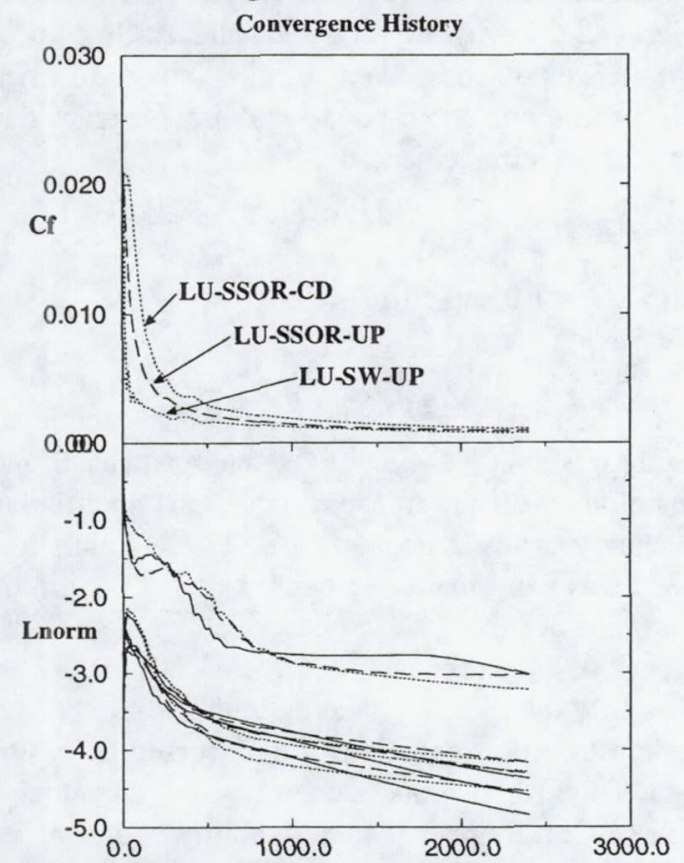

Figure 1: Typical Convergence Characteristics of the LU Numerics

The turbulence transport equations are solved uncoupled from the mean equations in order to reduce the numerical complexity. Therefore, the implicit operators needed to solve the additional two equations do not increase the rank of the overall implicit operator matrix for the mean solver. Thus, the time marching of the turbulence transport equations lags the mean equations marching by one iteration. This decoupling is not a major problem because the link between the mean turbulence equations is weak and occurs only through the diffusion terms. While the decoupling greatly simplifies the algorithm, some extra iterations may be required to resolve any sudden changes in the numerical solutions. Furthermore, it is possible that this can cause numerical instabilities during early stages of the computation, when rapid changes in the solution are expected. In order to minimize this effect, the source interaction term has been implicitly formulated and the iteration process was started from CFL of 1.0 or less.

\section{Numerics}

The governing equations are transformed into the following form when the body fitted coordinate system is used:

$$
\frac{\partial \hat{Q}}{\partial t}+\frac{\partial}{\partial \xi}\left(\hat{F}-\hat{F}_{v}\right)+\frac{\partial}{\partial \eta}\left(\hat{G}-\hat{G}_{v}\right)=\hat{H}
$$

where

$$
\begin{array}{rl}
Q=h Q & H=h H \\
\hat{E}=h\left(\xi_{x} F+\eta_{x} G\right) & \hat{E}_{v}=h\left(\xi_{x} F_{v}+\eta_{x} G_{v}\right) \\
\hat{G}=h\left(\xi_{v} F+\eta_{v} G\right) & \hat{G}_{v}=h\left(\xi_{v} F_{v}+\eta G_{v}\right)
\end{array}
$$

The delta form of Equation 8 can be written in the following implicit for:,

$$
\begin{gathered}
L S^{-1} U \Delta \hat{Q}=\Delta t R H S \\
L=\left(I+\Delta t\left(D_{\xi}^{-} \hat{A}^{+}+D_{\eta}^{-} \hat{B}^{+}-\hat{A}^{-}-\hat{B}^{-}-\hat{T}\right)\right) \\
U=\left(I+\Delta t\left(D_{\xi}^{-} \hat{A}^{-}+D_{\eta}^{-} \hat{B}^{-}-\hat{A}^{+}-\hat{B}^{+}\right)\right) \\
S=\left(I+\Delta t\left(\hat{A}^{+}+\hat{B}^{+}-\hat{A}^{-}-\hat{B}^{-}\right)\right)
\end{gathered}
$$

where the explicit right handside(RHS) is defined as the following,

$$
R H S=\left(D_{\xi}\left(\hat{F}-\hat{F}_{v}\right)+D_{\eta}(\hat{G}-G v)\right)+\hat{H}
$$

and $\Delta \mathrm{Q}$ is defined as $\mathrm{Q}^{\mathrm{n}+1}-\mathrm{Q}^{\mathrm{n}}$.

The source $\operatorname{term}(H)$ contains the effect of turbulent energy transfer between the dissipative scales and energy containing scales as well as some of the low Reynolds effects. Therefore, proper numerical treatment of these terms is essential in turbulent flow solver development. In near-wall flow situations, this term tends to get extremely stiff and therefore in most cases the implicit representation of this term is essential in maintaining numerical stability. This difficulty is further extenuated when the turbulence model is expected to handle a rapid exchange in turbulent energy between the dissipative scales and the turbulent energy scales due to externally generated gradients such as shock-waves. Similar observations have been made by other investigators. ${ }^{15,20}$ However, the exact formulation of the source flux Jacobian term varies from study to study. Initially, we have used the following $2 \times 2$ matrix for the source Jacobian on the left hand side of the Equation 9:

$$
T_{i j}=\left[\begin{array}{cc}
-\left(\frac{P_{k}}{\rho k}\right)-\frac{\varepsilon}{k} & \frac{2 P_{k}}{\rho \varepsilon} \\
-C_{\varepsilon 1}\left(\frac{\varepsilon}{k}\right)^{2}\left(\frac{P_{k}}{\rho \varepsilon}-f_{\varepsilon} \frac{C_{\varepsilon 2}}{C_{\varepsilon 1}}\right) & C_{\varepsilon 1} \frac{\varepsilon}{k}\left(\frac{P_{k}}{\rho \varepsilon}-2 f_{\varepsilon} \frac{C_{\varepsilon 2}}{C_{\varepsilon 1}}\right)
\end{array}\right]
$$

Huang and Coakley ${ }^{15}$ showed that the source Jacobian should be formulated so that the overall numerical scheme maintains a positive left hand side. We have found from our experience that this particular point is not 
essential for the SSOR technique because of the diagonal dominance generated by the maximum spectral radii. However, for the SW technique, maintaining a positive left hand side is more important because the local spectral radii do not always dominate. In order to maintain a positive left handside, only the negative diagonal terms of $T_{i j}$ were used along with the SW algorithm.

\section{III.a LU-SSOR}

In the Symmetric Successive Over Relaxation numerical technique, the global eigenvalue, directional splitting, and LU decompositions are used to set-up the left hand side(LHS) of Equation 8 such that only scalar inversions are required to solve the equation. The flux Jacobians $\mathrm{A},{ }^{+} \mathrm{A},{ }^{-} \mathrm{B},{ }^{+}$and $\mathrm{B}^{-}$are constructed such that the eigenvalues of $\mathrm{A}^{+}$and $\mathrm{B}^{+}$are positive and the eigen values of $\mathrm{A}^{-}$and $\mathrm{B}^{-}$are negative. The convergence is achieved through a four step sweeping process:

$$
\begin{gathered}
\Delta \hat{Q}_{i j}^{I}=\Delta t\left(\left(I+\Delta t\left(\frac{\gamma_{A}}{\Delta \xi}+\frac{\gamma_{B}}{\Delta \eta}\right)\right)\right) R H S+\frac{\Delta t}{\Delta \xi} \hat{A}_{i-1, j}^{+} \Delta \hat{Q}_{i-1}^{I} \\
\left(I+\Delta t\left(\frac{\gamma_{A}}{\Delta \xi}+\frac{\gamma_{B}}{\Delta \eta}\right)-\Delta t T_{i j}\right) \Delta \hat{Q}_{i j}^{I I}= \\
\Delta \hat{Q}_{i j}^{I}+\frac{\Delta t}{\Delta \eta} \hat{B}_{i, j-1}^{+} \Delta \hat{Q}_{i, j-1}^{I I} \\
\Delta \hat{Q}_{i j}^{I I I}=\Delta \hat{Q}_{i j}^{I I}-\frac{\Delta t}{\Delta \xi} \hat{A}_{i+1, j}^{-} \Delta \hat{Q}_{i+1, j}^{I I I} \\
\left(I+\Delta t\left(\frac{\gamma_{A}}{\Delta \xi}+\frac{\gamma_{B}}{\Delta \eta}\right)\right) \Delta \hat{Q}_{i j}=\Delta \hat{Q}_{i j}^{I I I}-\frac{\Delta t}{\Delta \eta} \hat{B}_{i, j+1}^{-} \Delta \hat{Q}_{i, j+1}
\end{gathered}
$$

where the split flux Jacobians are defined as,

$$
\hat{A}^{ \pm}=\frac{1}{2}\left(\hat{A} \pm \gamma_{A} I\right)
$$

and $\mathrm{B}+, \mathrm{B}-$ are similarly defined. Here, the spectral radii are corrected by the viscous terms $M_{\xi} N / \Delta \xi^{2}$ and $M_{\eta} N / \Delta \eta^{2}$.

\section{III.b LU-SW}

In order to improve the convergence of skin friction prediction, flux vector splitting procedure of Steger and Warming ${ }^{23}$ was also studied. This decomposition is achieved by using local eigenvalues based on local characteristic wave speeds. This approach yields simple rank two matrix operations for the de-coupled numerical formulation used in this study. The explicit terms on the right hand side of Equation 8 were differenced using the van Leer's flux-vector upwind differencing technique to maintain numerical accuracy. Typical Jacobian matrices split according to the local wave speeds are as follows

$$
\lambda_{A}=\left[\begin{array}{ll}
U & 0 \\
0 & U
\end{array}\right] \quad \hat{A}^{ \pm}=\frac{\left(\lambda_{A} \pm\left|\lambda_{A}\right|\right)}{2}
$$

and $\lambda_{\mathrm{b}}$ as well as $\mathrm{B}^{+}, \mathrm{B}^{-}$are similarly defined.

When a directional splitting process similar to the LU-SSOR is used, Equation 10 reduces to the following four step procedure:

$$
\begin{gathered}
\Delta \hat{Q}_{i j}^{I}=\Delta t R H S+\frac{\Delta t}{\Delta \xi}\left(\hat{A}^{+}+\frac{M_{\xi} N}{\Delta \xi}\right)_{i-1, j} \Delta \hat{Q}_{i-1, j}^{I} \\
\left(I+\Delta t\left(\frac{|\hat{A}|}{\Delta \xi}+\frac{|\hat{B}|}{\Delta \eta}+\frac{M_{\xi} N}{\Delta \xi^{2}}+\frac{M_{\eta} N}{\Delta \eta^{2}}\right)-\Delta t T_{i j}\right) \Delta \hat{Q}_{i j}^{I I}=\Delta \hat{Q}_{i j}^{I} \\
+\frac{\Delta t}{\Delta \eta}\left(\hat{B}^{+}+\frac{M_{\eta} N}{\Delta \eta}\right) \hat{B}_{i, j-1}^{-} \Delta \hat{Q}^{I I}{ }_{i, j-1} \\
\Delta \hat{Q}_{i j}^{I I I}=\left(I+\Delta t\left(\frac{|\hat{A}|}{\Delta \xi}+\frac{|\hat{B}|}{\Delta \eta}\right)\right) \Delta \hat{Q}_{i j}^{I I} \\
\left.\left(I+\Delta t\left(\frac{|\hat{A}|}{\Delta \xi}+\frac{|\hat{B}|}{\Delta \eta}+\frac{M_{\xi} N}{\Delta \xi^{2}}+\frac{M_{\eta} N}{\Delta \eta^{2}}\right)\right) \Delta \hat{Q}_{i j}^{-}=\frac{M_{\xi} N}{\Delta \xi}\right)_{i+1, j} \hat{Q}_{i+1, j}^{I I I} \\
-\frac{\Delta t}{\Delta \eta}\left(\hat{B}_{i j}^{-}-\frac{M_{\eta} N}{\Delta \eta}\right)_{i, j+1}^{I I} \quad \Delta \hat{Q}_{i, j+1}
\end{gathered}
$$

Here the viscous terms in thin-layer form have been added to the left handside to maintain stability in the near wall regions of the computation.

\section{III.c Van Leer's MUSCL Upwinding Technique}

In order to obtain upwind numerical accuracy, the flux vector of the inviscid terms in the RHS are evaluated based on the Monotone Upwind-Centered Scheme for Conservative Law (MUSCL) ${ }^{24}$ approach given by the following:

$$
\begin{gathered}
\frac{\partial F}{\partial \xi}=\left(F^{+}\left(Q^{-}\right)+F^{-}\left(Q^{+}\right)\right)_{L}-\left(F^{+}\left(Q^{-}\right)+F^{-}\left(Q^{+}\right)\right)_{R} \\
F^{\mp}=\frac{1}{4} \rho \sqrt{\bar{\gamma} R T}(M \pm 1)^{2} Q
\end{gathered}
$$

where non-conservative variables $\mathrm{Q}(\mathrm{k}, \varepsilon)$ are evaluated using the MINMOD flux limiter approach proposed by van 
Leer(Hirsh ${ }^{22}$ ). We have used a second order scheme for all of the computations.

\section{Boundary and Initial Conditions}

Zero gradient conditions were assumed at the freestream boundaries of the computation. At the wall surfaces, zero boundary conditions were used in the lowReynolds number $\mathrm{k}-\varepsilon$ model of $\mathrm{Chien}^{18}$ and LaunderSharma. ${ }^{17}$ Boundary conditions based on the near-wall Kolmogorov scales were used in the $\mathrm{k}-\varepsilon$ model proposed by Shih. ${ }^{19}$ The wall turbulent kinetic energy and dissipation are defined as follows:

For Chien and Launder-Sharma Models,

$\mathrm{k}_{\mathrm{bc}}=0.0$, and $\varepsilon_{\mathrm{bc}}=0.0$

For Shih and Lumley Model,

$\rho k_{b c}=0.25 \rho_{w} U_{\tau}^{2}$, and $\rho \varepsilon_{b c}=0.251 \rho_{\mathrm{w}}{ }^{2} \mathrm{U}_{\tau}{ }^{4} / \mu_{\mathrm{w}}$

The inlet velocity profile was developed from the initial value of the skin friction coefficient, boundary-layer thickness and the law of the wall assumption. The Van-Driest transformation ${ }^{25}$ was used to determine the velocity profile from the assumed loglaw profile. The thermal profile was developed using a typical Crocco's parabolic relationship. The turbulence kinetic energy and dissipation profiles were developed from the velocity profile, along with the equilibrium constraints and the assumed length scale profile:

Typical law of the wall formulation can be written in the following non-dimensional form,

$$
U^{+}=\frac{1}{0.41} \ln \left(y^{+}\right)+5.1+\frac{\Pi}{\delta} W\left[\frac{y}{\delta}\right]
$$

where the function $W(y / \delta)$ is the wake correction. The transformation proposed by Van Driest is then used to find the actual velocity profile which includes the effect of density from the log-law. A typical transformed equation for the arbitrary temperature ratio can be written in the following form:

$$
\begin{array}{r}
\left.\frac{u}{U_{e}=\frac{\sqrt{b^{2}+4 a}}{2 a} \sin \left(U+\sqrt{\frac{a C_{f} T_{w}}{2 T_{e}}}\right.}-\operatorname{asin}\left(\frac{b}{\sqrt{b^{2}+4 a}}\right)\right) \\
+\frac{1}{2 a}\left[\frac{T_{e}}{T_{w}}+a-1\right]
\end{array}
$$

where

$$
a=\frac{T_{e}}{T_{w}} \bar{r} \frac{(\bar{\gamma}-1)}{2} M_{e}^{2} \quad b=\frac{T_{e}}{T_{w}}+a-1
$$

This generalized transformation reduces to a simpler form derived by White ${ }^{26}$ when flow is assumed to be adiabatic $\left(\mathrm{T}_{\mathrm{aw}} / \mathrm{T}_{\mathrm{w}}=1.0\right)$. Furthermore, the velocity profile is determined from this relationship given only the initial skin friction coefficient, boundary-layer thickness, and the temperature ratio. The thermal profile is developed from the modified Crocco-Cohen relationship, which is general enough to satisfy both cold wall and adiabatic wall boundary conditions. The Crocco-Cohen relationship of total enthalpy ratio has been shown by Wallace ${ }^{27}$ to be:

$\frac{\left(H-H_{w}\right)}{\left(H_{e}-H_{w}\right)}=\left(1-\frac{C_{t}}{\left(1-\frac{H_{e}}{H_{w}}\right)}\right)\left[\frac{u}{U_{e}}\right]+\frac{C_{t}}{\left(1-\frac{H_{e}}{H_{w}}\right)}\left[\frac{u}{U_{e}}\right]^{2}$

where the parameter $\left(\mathrm{C}_{\mathrm{t}}\right)$ ranges from 0 , for CroccoBusemann linear formulation, to $1-\mathrm{H}_{\mathrm{e}} / \mathrm{H}_{\mathrm{w}}$, for a parabolic formulation which corrects for heat dissipation $(\operatorname{Pr}=1.0)$. This relationship can be further simplified by expanding the total enthalpy expression to the static temperature ratios. This simplification leads to the following expression for the non dimensional static temperature,

$$
\frac{T}{T_{w}}=1.0+\bar{g}\left[\frac{u}{U_{e}}\right]+\bar{h}\left[\frac{u}{U_{e}}\right]^{2}
$$

$$
\begin{gathered}
\bar{g}=\left(1-C_{t}\right)\left[\left(1+\bar{r} \frac{(\bar{\gamma}-1)}{2} M_{e}^{2}\right) \frac{T_{e}}{T_{w}}\right]-1.0 \\
\bar{h}=\frac{T_{e}}{T_{w}}\left[C_{t}\left(1.0+\bar{r} \frac{(\bar{\gamma}-1)}{2} M_{e}^{2}\right)-\bar{r} \frac{(\bar{\gamma}-1)}{2} M_{e}^{2}\right]
\end{gathered}
$$

Thus, once the velocity profile is developed from the previously derived velocity formulation the thermal profile can be completely developed from the above expression. Finally, the turbulent kinetic energy and dissipation rate profiles were developed from the equilibrium $\left(\mathrm{P}_{\mathrm{k}}=\rho \varepsilon\right)$ assumption and the freestream condition constraints.

$$
k=\left[\frac{\partial U}{\partial y}\right]^{2} \frac{l^{2}}{C_{\mu}^{1 / 2}}+k_{\infty} \frac{y}{\delta} \quad \varepsilon=C_{\mu}^{3 / 4} \frac{k^{3 / 2}}{l}+\varepsilon_{\infty} \frac{y}{\delta}
$$

where the length scale is assumed to be

$$
l=\min (0.4 y, 0.085 \delta)
$$


The freestream turbulent kinetic energy and dissipation are determined from the following simple relationships, which maintain reasonable freestream turbulence and eddy viscosity levels:

$$
k_{\infty}=\frac{3}{2}\left(T_{u} U_{\infty}\right)^{2} \quad \varepsilon_{\infty}=C_{\mu} \frac{k^{2}}{v_{\infty}}
$$

\section{Test Cases}

Several supersonic turbulent boundary-layers with or without pressure gradients were analyzed to validate numerical implementation of the baseline turbulence models. Figure 2 shows typical Mach 2.87 inlet velocity and temperature profiles. These profiles have been developed by the method described previously to satisfy the experimental Reynolds number and the boundary-layer thickness. Initially, the profiles were computed a location upstream of the measurement station. Then the zeropressure gradient boundary-layer problem was solved to find fully developed boundary-layer velocity and temperature profiles. These profiles are then used as the inlet conditions for the Navier-Stokes/turbulence-model computations. Typically, a boundary thickness of $0.025 \mathrm{~m}$ and skin friction coefficient of 0.0013 have been used to develop the inlet profiles. A turbulence intensity of 1 percent and an adiabatic wall have been assumed.

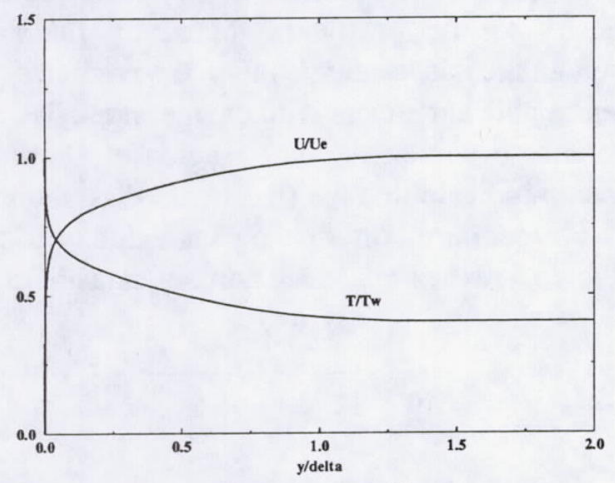

Figure 2: Inlet Profiles

A Mach 2.87 flow with freestream Reynolds number of $6.3 \times 10^{7} / \mathrm{m}$ was used to match the test condition of experimental studies of Smits et al. ${ }^{28,29}$ A typical grid system used to resolve a zero-pressure gradient turbulent boundary layer is shown in Figure 3. The overall height of the grid system is usually three boundary-layer thicknesses. The length of the computational domain varied from 0.25 $\mathrm{m}$ to $1.00 \mathrm{~m}$. Uniform grid distribution is usually used in the streamwise direction. Typically, an 81 by 63 grid system was used for the shock-wave/boundary-layer interaction prediction and a 101 by 63 grid system was used in the flat plate boundary-layer predictions. The vertical spacing of this grid system has been exponentially stretched to maintain a near-wall spacing of approximately 1.0 in nondimensional coordinate $\left(y^{+}\right)$. Approximately, 45 of 63 points are located in a boundary-layer region. The zero gradient boundary condition has been used in the exit and freestream plane of the grid system. The inlet profiles of velocity, temperature and pressure are fixed.

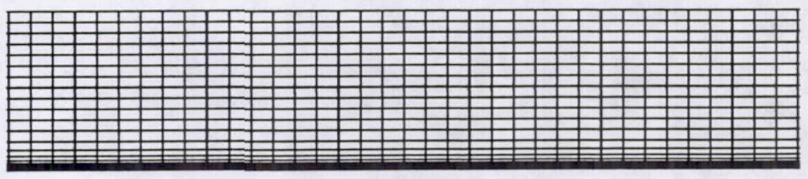

Figure 3: A Typical Grid System for Zero Pressure Gradient Turbulent Boundary-layer Predictions

Typical convergence behavior of the turbulent quantities along with the skin friction coefficient time history, obtained using a constant CFL variable time step marching technique, is shown in Figure 4. Convergence was determined by a two to three order reduction in the global residual of all quantities and reduction of the skin friction level to the third significant figure. This figure once again shows that the SSOR convergence is slower than the $\mathrm{SW}$ convergence. Although the SSOR numerical technique is stable to infinitely large CFL numbers, only values near one have been used to yield more meaningful comparisons to the SW flux vector splitting technique. This figure shows that SSOR technique with central difference RHS required more than 2000 iterations as compared to the approximately 1000 iterations required for the SW technique.

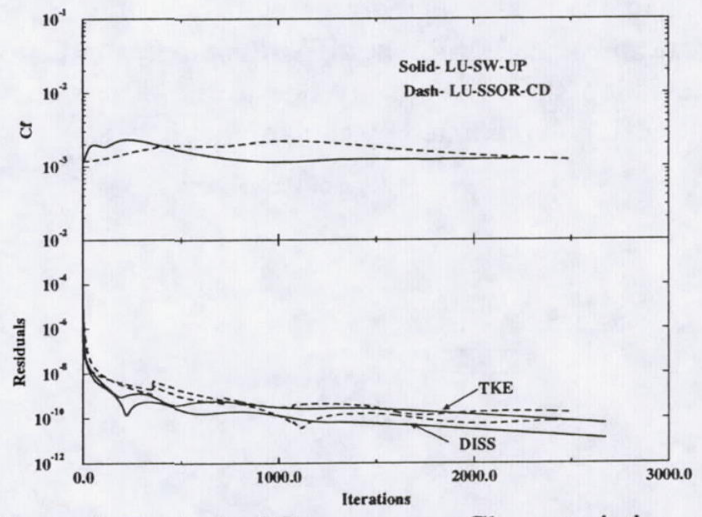

Figure 4: Typical of Convergence Characteristics

Figure 5 shows the typical skin friction coefficient comparisons between the model predictions and the experimental data reported by Smits et al. Although all of the model computations were started from the same inlet condition, all three models responded differently. The differences in the initial skin friction level can be as much as 60 percent. However, the skin friction differences relax into a more acceptable level down stream of the inlet, where the variation in the skin friction level reduced to 
approximately 15 percent. The transition like behavior shown in this figure has been forced by not including the production contribution of the turbulence model in the first few stations of computation which force the solution to be laminar. Figure 5 also shows that Chien's model prediction of the skin friction is the lowest of the three model predictions.

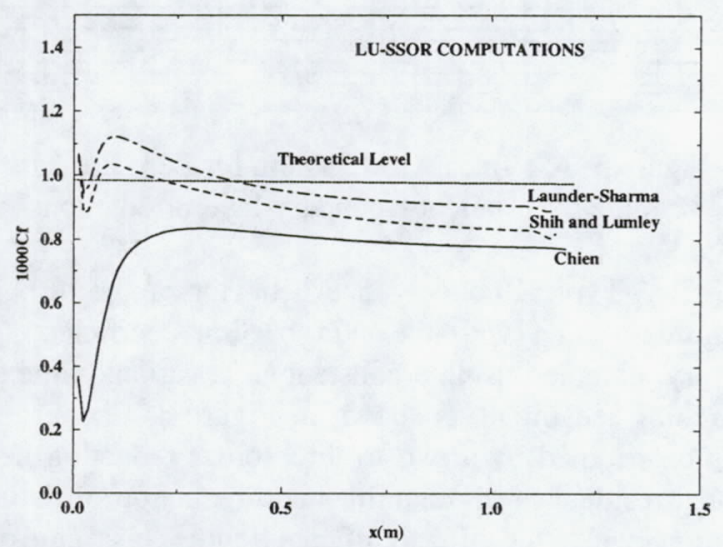

Figure 5: Skin Friction Distribution Mach 2.87 Turbulent Boundary-Layer

The law of wall profile predictions are compared in Figure 6. All profiles shown in Figure 6 have been transformed into equivalent velocity form using the VanDriest transformation to eliminate the effects of variable density. Although the prediction made using the ShihLumley and Launder-Sharma models seem to be in closest agreement with the experimental data, all of the predictions are in reasonable agreement with both the experimental data and an earlier Reynolds Stress model prediction. ${ }^{8}$ The deviation shown is mostly due to the discrepancy in the skin friction predicted by each of the models.

\section{Law of Wall Profile}

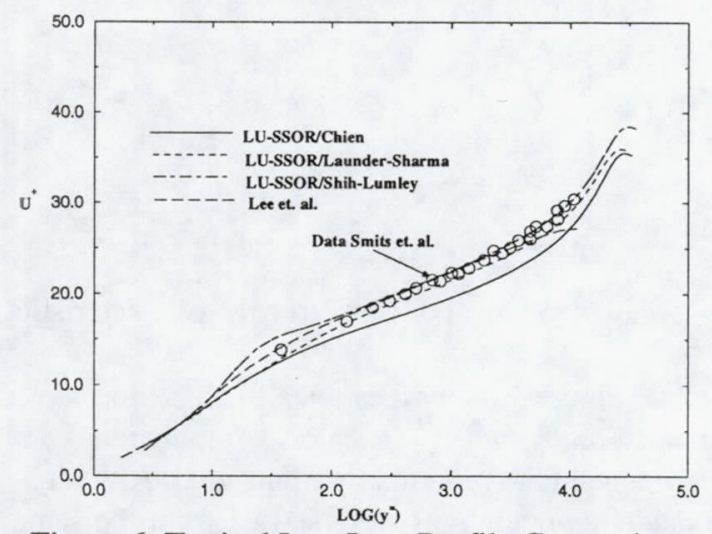

Figure 6: Typical Log-Law Profile Comparisons

Typical profiles of the normal Reynolds Stresses $\left(\tau_{x x} \tau_{y y}\right)$ are shown in Figure 7 . The Reynolds Stresses in this figure are normalized by the friction velocity. Note that the agreement between the experimental data and the prediction is only reasonable. Furthermore, because of the isotropic assumption, $\mathrm{k}-\varepsilon$ model prediction of $\tau_{\mathrm{xx}}$ is equal to $\tau_{\mathrm{yy}}$. This is not supported by the experimental data. This is consistent with the earlier observation of Lee. ${ }^{9}$ Either an anisotropic eddy viscosity k$\varepsilon$ model or a full Reynolds Stress model would be required to correctly model the normal stresses. Despite this difficulty, the other parameters of interest, such as skin friction coefficient, are predicted correctly. This is in part due to the damping factors employed by the $\mathrm{k}-\varepsilon$ model which mask such deficiencies.

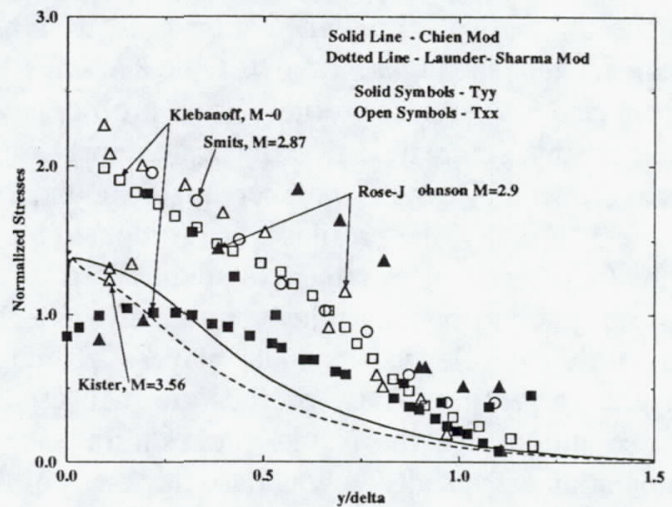

Figure 7: Non-Dimensional Normal Stress $\left(\tau_{x x} \tau_{y y}\right)$

Figure 8 compares the measured kinematic eddy viscosity to the profile predicted by the $\mathrm{k}-\varepsilon$ models. Although the data scatter is large, the predicted profiles are in reasonable agreement with the measured data. The major difference occurs in the outer region of the profile which seems to indicate that the freestream dissipation used may be too large. Furthermore, the peak value of eddy viscosity predicted and the high Mach number data are in reasonable agreement.

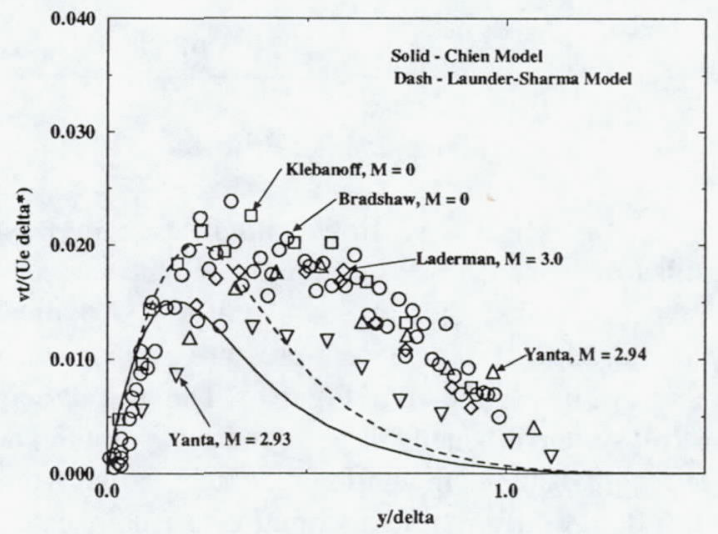

Figure 8: Typical Eddy Viscosity Profile

A more complex problem of interest is the viscous-inviscid interaction caused by a shock-wave impinging on a turbulent boundary-layer. As shown later, the turbulence models seem unable to correctly handle 
near-wall relaxation behavior caused by external pressure gradients. This is an important flow feature that contributes significantly to the flow losses in complex high speed combustors $^{30}$ and to other external aerodynamic parameters. ${ }^{31}$ Therefore, accurate modeling of this problem is a primary requirement for any generalized CFD method developed for internal flow analysis. In order to study this class of problem in more detail and to perform a more rigorous test of the turbulence model-numerics combination developed, several cases of the viscous interaction problem experimentally studied by Smits et al. have been computed. In this flow configuration the shockwave is generated by a wedge ramp The shock-wave then interacts with the incoming turbulent boundary-layer, leading to separation at the higher pressure ratio conditions.

Turbulence model predictions of 8 and 24-degree ramp angle conditions are compared with the experimental data of Smits et al. ${ }^{28,29}$ For the 8 degree case, the rise in pressure was high enough to cause only a thinning of the near wall boundary-layer, and not a separation. However, for the 24 degree case, the turbulent boundary-layer nearwall is fully separated. The separation makes the 24 degree cases a more difficult test for the turbulence models. A typical grid system used to model this flowfield is illustrated in Figure 9. The incoming boundary-layer for all of the predictions have been generated using Chien model. Typically, a flat plate region $0.05 \mathrm{~m}$ and $0.15 \mathrm{~m}$ ahead of the corner has been resolved in the 8 degree case and 24 degree case, respectively. The wedge length is $0.2 \mathrm{~m}$, and a uniform grid distribution is used in the streamwise direction. The total height of the computational domain is three times the boundary-layer thickness. The vertical spacing of this grid system has been exponentially stretched to maintain a near-wall spacing of approximately 1.0 in non-dimensional coordinate $\left(\mathrm{y}^{+}\right)$at the inlet of the computational domain.

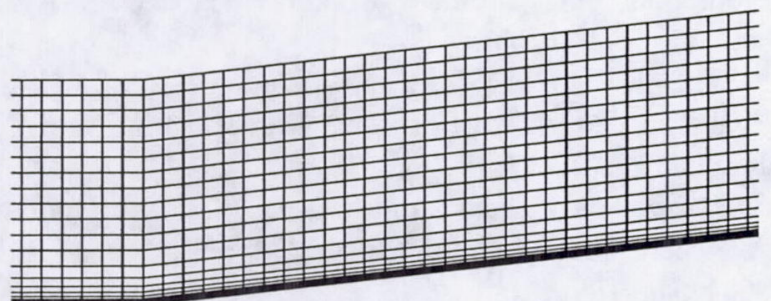

Figure 9: Typical Flat plate-Wedge Grid System

Figure 10 compares the predicted wall pressure and skin friction distributions and the experimental data of Smits et al. for the 8 degree case. This figure shows that the actual turning angle of the incoming turbulent boundarylayer is reasonably captured by all of the k- $\varepsilon$ models, which results in close agreement of wall pressure predicted and measured. However, the prediction of the skin friction is more difficult. The relaxation of the skin friction is over predicted by Chien model and is under predicted by the Launder-Sharma model.

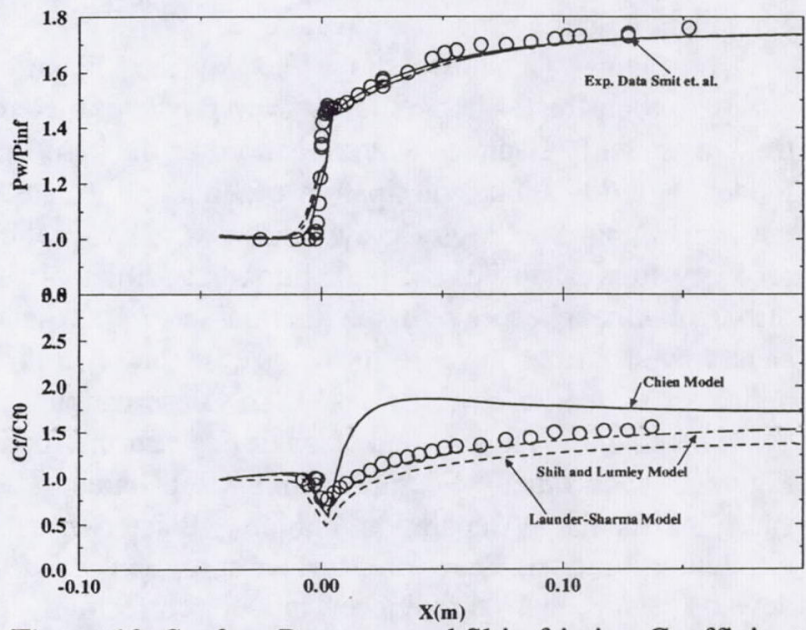

Figure 10: Surface Pressure and Skin friction Coefficient Distribution for 8 degree Ramp case

Figure 11 compares the typical wall pressure and skin friction distributions predicted and the experimental data of Smits et al. for the 24 degree case. The presence of a strong shock-wave causes the boundary-layer to separate and to form a separation bubble near the corner of the compression ramp. The separation bubble then causes the inviscid turning well ahead of the wedge which leads to the two step wall pressure distribution. This early turning causes flow to developed a "lambda" shock structure with a pressure "plateau." ${ }^{31}$ This flow structure has been predicted by all of the $k-\varepsilon$ models. The total rise in wall pressure has also been correctly predicted by all of the models. However, the predicted size of the bubble and the

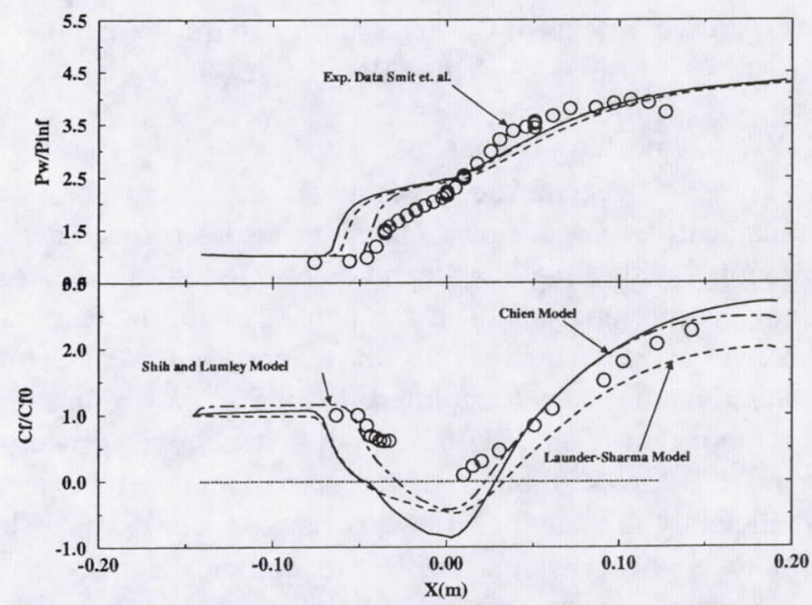

Figure 11: Surface Pressure and Skin friction Coefficient Distribution for 24 degree Ramp case

strength of the wall skin-friction recovery vary among the models. Both the Launder-Sharma model and the Chien model over predicted the downstream skin-friction 
recovery and the size of the separation bubble. The ShihLumley model prediction of the separation bubble is in best agreement with the experimental data.

Figure 12 shows the effect of the dilatation dissipation on the 24-degree ramp flow prediction. Here, the $\mathrm{k}-\varepsilon$ model of Launder-Sharma is used as the base line model. The dilatational dissipation of Equation 7 causes the separation bubble to grow and wall skin-friction recovery to weaken(i.e. $\varepsilon=\varepsilon+\varepsilon_{d}$ ). Further addition of the turbulent kinetic energy to the thermal energy balance causes even further increase in the size of the separation bubble predicted(i.e. $\mathrm{E}=\mathrm{C}_{\mathrm{v}} \mathrm{T}+\overline{\mathrm{U}}^{2}+\mathrm{k}$ ). This observation is in agreement with Huang and Coakley. ${ }^{15}$ Furthermore, Figure 12 shows that the compressibility terms are not the cause of the large separation bubble predicted by the baseline $\mathrm{k}-\varepsilon$ model. Therefore, further study is needed to develop additional turbulence model corrections to improve separation bubble size predictions.

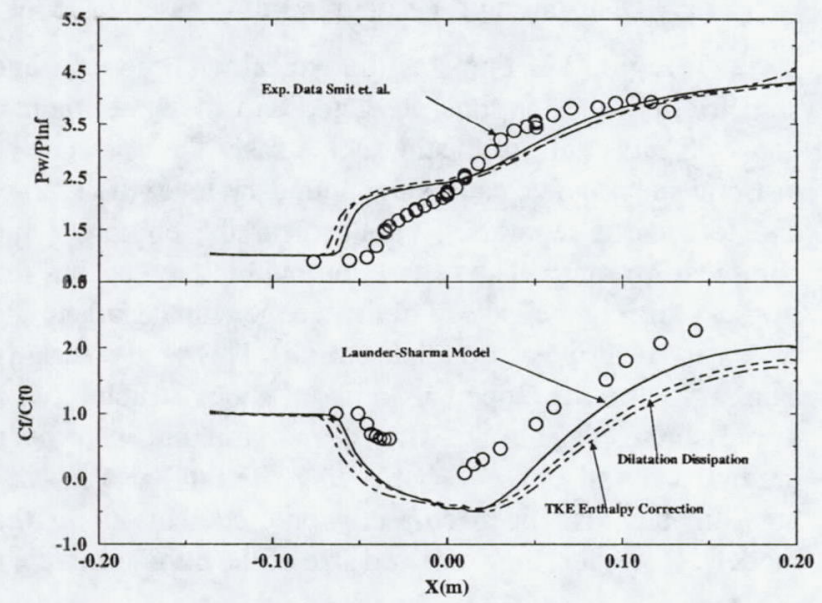

Figure 12: Effects of Compressibility on the Prediction of 24-degree Ramp case

\section{Concluding Remarks}

A generalized flow solver using Lower-Upper diagonal decomposition techniques has been successfully coupled with three low-Reynolds number $\mathrm{k}-\varepsilon$ models for engineering analysis. The limitations of the models and convergence characteristics in several supersonic flow situations have been explored. We have shown, that the combination of the LU based implicit numerical method and the low-Reynolds number $\mathrm{k}-\varepsilon$ model can be used efficiently in studying problems ranging from simple flat plate boundary characteristics to complex shock-wave/ boundary-layer interaction. In this study we have also shown that an SSOR marching process is slowest in obtaining a steady state solution; and upwinding the inviscid terms tend to improve the efficiency. Furthermore, using the Steger-Warming flux vector formulation with the explicit terms upwinded seems to be optimal for choice for an LU based turbulence model solver. All three turbulence models are able to predict a zero pressure gradient supersonic turbulent boundary-layer with reasonable accuracy. However, the more complex problem of a shockwave/boundary-layer interaction is handled less adequately by these models. The wall skin friction recovery behaviors predicted are drastically different among the models. It is found that for the 8 and 24 degree shock-wave/boundarylayer interaction studied, Launder-Sharma model and ShihLumley model predictions are closest to the experimental data. It was also found that the optimal CFL number which is allowed for both these model is approximately 1.0 or less for the Launder-Sharma and Shih and Lumley model while the allowable CFL number for the Chien model can be up to 5. The effects of compressibility, in the form of dilatation dissipation, increase the separation bubble size and reduce the wall skin-friction level.

\section{Acknowledgment}

This work was funded by Contract NAS 3-25266 monitored by Dr. D. R. Reddy and Dr. L. A. Povinelli. Extremely helpful suggestions of Dr. G. Huang of NASA Ames RC. are gratefully acknowledged.

\section{References}

1. Lee, J., "A Numerical Investigation of Mixing in Supersonic Combustor with Hypermixing Injectors," AIAA J. of Propulsion and Power, March 1994.

2. Johnson D. A., and King L. S.,"A Mathematically Simple Turbulence Closure Model for Attached and Separated Turbulent Boundary-Layers," AIAA J., Vol. 23, No. 11, Sept. pp. 1684-1692.

3. Jones W. P., and Launder B. E., "The Prediction of Laminarization with a Two-Equation Model of Turbulence," Int. J. of Heat and Mass Transfer, Vol. 15, 1972, pp. 301-314.

4. Saffman D. G., and Wilcox D., "Turbulence Model Predictions for Turbulent Boundary-Layers," AIAA J., Vol. 12, pp. 541-546.

5. Speziale C. G., Abid, R., and Anderson E. C., "A Critical Evaluation of Two-Equation Models for Near-Wall Turbulence," AIAA P 90-1481.

6. Hytopoulos, E., and Simpson R.L., "Critical Evaluation of Recent Second-Order Closure Models," AIAA P 930081. Jan. 1990.

7. Launder B.E., and Shima N., "Second Moment Closure for the Near-Wall Sublayer: Development and Application," AIAA J., Vol. 27, No.10, Oct 1989, pp.13191325 .

8. Lee J., Taulbee D. B., and Holden M. S., "Study of Turbulence on Supersonic Compression Surface using Reynolds Stress Model," AIAA J. Vol. 30, No. 7, July 1992, pp. 1738-1746.

9. Patel V. C., Rodi W., and Scheuerer G., "Turbulence Models for Near-Wall and Low Reynolds Number Flows: 
A Review," AIAA J., Vol. 23, No. 9, 1985, pp.1308-1319. 10. Coakley T. J., and Huang, P. G., "Turbulence Modelling for High Speed Flows," AIAA Paper 92-0436, Jan 1992.

11. Michelassi V., and Shih T. H., "Elliptic Flow Computation by Low Reynolds Number Two-Equation Models," NASA TM 105237, 1991.

12. Zeman O., "A New Model for Super/Hypersonic Turbulent Boundary Layers," AIAA P 93-0897.

13. Sarkar, S. G., Erlebacher, M.Y. Hussani, and H.O. Kreiss, "The Analysis and Modeling of Dilatational Terms in Compressible Turbulence," JFM Vol. 227, pp473-483.

14. Taulbee D. B., Lee J., and VanOsdal J., "Modeling of Pressure Dilatations in Compressible Flows," 43rd. Physical Society Meeting, Ithacca, NY.

15. Huang P. G., and Coakley, T. J., "An Implicit NavierStokes Code for Turbulent Flow Modeling," AIAA Paper 92-0547, Jan. 1992.

16. Favre A., "Equations of Compressible Turbulent Gases, General Formulation," Journal of Mechanics, Vol. 4, 1965, pp.391-421.

17. Launder B. E., and Sharma B. I., "Application of Energy-Dissipation Model of Turbulence to the Calculation of Flow near a Spinning Disc," Letters in Heat and Mass Transfer, Vol. 1, 1974,pp.131-138.

18. Chien K.Y., "Prediction of Channel and BoundaryLayer Flows with a Low-Reynolds Number Turbulence Model," AIAA J., Vol. 20, No. 1, 1982, pp.33-38.

19. Shih T. H., and Lumley J. L., "Kolmogorov Behavior of Near-Wall Turbulence and its Application in Turbulence Modelling," NASA TM 105663, 1992.

20. Gerolymos G. A., "Implicit Multiple-Grid Solution of the Compressible Navier-Stokes Equations Using k- $\varepsilon$ Turbulence Closure," AIAA J. Vol. 28, No. 10, Oct. 1990. 21. Wilcox D., "Progress in Hypersonic Turbulence Modeling," AIAA P 91-1785.

22. Jameson A., and Yoon S., "Lower-Upper Implicit Schemes with Multiple Grids for Euler Equations," AIAA J., Vol. 25, July 1987, pp.929-935.

23. Steger J. L, and Warming R. F., " Flux Vector Splitting of the Inviscid Gas Dynamics Equations with Applications to Finite Difference Methods," J. of Comp. Physics, Vol. 40., No. 2, Apr. 1981, pp. 283.

24 Hirsch, C., Numerical Computations of Internal and External Flows, 1990.

25. Van Driest E. R., "Turbulent Boundary-Layers in Compressible Fluids," J. of Aero., Vol. 18, March 1951, pp.145-160.

26. White F. M., Viscous Flow, McGraw Hill, New York, 1971.

27. Wallace J. E., "Hypersonic Boundary-Layer Studies at Cold Wall Conditions," Proceedings of 1967 Heat Transfer and Fluid Mechanics Institute, P. Libby ed.

28. Degani D., and Smits A. J., "Effect of Short Regions of
Surface Curvature on Compressible Turbulent Boundary Layers," AIAA J. Vol. 26. No.1 Jan 1990, pp 113-119. 29. Smits A. and Muck C., "Experimental Study of Three Shock Wave/Boundary Layer Interactions," JFM, Vol 182, Sept. 1987, pp291-314.

30. Riggins D. W., and McClinton C. E. "A Computational Investigation of Flow Losses in a Supersonic Combustor," AIAA P 90-2093.

31. Holden M. S., "A Review of Aerothermal Problems Associated with Hypersonic Flight," AIAA P 86-0267. 
Public reporting burden for this collection of information is estimated to average 1 hour per response, including the time for reviewing instructions, searching existing data sources, gathering and maintaining the data needed, and completing and reviewing the collection of information. Send comments regarding this burden estimate or any other aspect of this collection of information, including suggestions for reducing this burden, to Washington Headquarters Services, Directorate for Information Operations and Reports, 1215 Jefferson Davis Highway, Suite 1204, Arlington, VA 22202-4302, and to the Office of Management and Budget, Paperwork Reduction Project (0704-0188), Washington, DC 20503.

\begin{tabular}{|l|l|l|}
\hline 1. AGENCY USE ONLY (Leave blank) & $\begin{array}{c}\text { 2. REPORT DATE } \\
\text { April } 1994\end{array}$ & $\begin{array}{r}\text { 3. REPORT TYPE AND DATES COVERED } \\
\text { Final Contractor Report }\end{array}$ \\
\hline
\end{tabular}

4. TITLE AND SUBTITLE

An Analysis of Supersonic Flows with Low-Reynolds Number Compressible

Two-Equation Turbulence Models Using LU Finite Volume Implicit

Numerical Techniques

6. $\operatorname{AUTHOR}(\mathrm{S})$

J. Lee

5. FUNDING NUMBERS

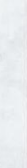

7. PERFORMING ORGANIZATION NAME(S) AND ADDRESS(ES)

Sverdrup Technology, Inc.

Lewis Research Center Group

2001 Aerospace Parkway

Brook Park, Ohio 44142

9. SPONSORING/MONITORING AGENCY NAME(S) AND ADDRESS(ES)

National Aeronautics and Space Administration

Lewis Research Center

Cleveland, Ohio 44135-3191

WU-505-62-40

C-NAS3-25266

8. PERFORMING ORGANIZATION REPORT NUMBER

E-8701

10. SPONSORING/MONITORING AGENCY REPORT NUMBER

NASA CR-195302

AIAA 94-0193

\section{SUPPLEMENTARY NOTES}

Prepared for the 32nd Aerospace Sciences Meeting \& Exhibit sponsored by the American Institute of Aeronautics and Astronautics, Reno, Nevada, January 10-13, 1994. J. Lee (work funded by NASA Contract NAS3-25266), presently at NYMA, Inc., Engineering Services Division, 2001

Aerospace Parkway, Brook Park, Ohio 44142. Project Manager, D.R. Reddy, organization code 2610, NASA Lewis Research Center, (216) $433-8133$.

\section{2a. DISTRIBUTION/AVAILABILITY STATEMENT}

12b. DISTRIBUTION CODE

Unclassified - Unlimited

Subject Category 02

\section{ABSTRACT (Maximum 200 words)}

A generalized flow solver using an implicit Lower-upper (LU) diagonal decomposition based numerical technique has been coupled with three low-Reynolds number $k-\varepsilon$ models for analysis of problems with engineering applications. The feasibility of using the LU technique to obtain efficient solutions to supersonic problems using the $k-\varepsilon$ model has been demonstrated. The flow solver is then used to explore limitations and convergence characteristics of several popular two equation turbulence models. Several changes to the LU solver have been made to improve the efficiency of turbulent flow predictions. In general, the low-Reynolds number $k-\varepsilon$ models are easier to implement than the models with wall-functions, but require much finer near-wall grid to accurately resolve the physics. The three $k-\varepsilon$ models use different approaches to characterize the near wall regions of the flow. Therefore, the limitations imposed by the nearwall characteristics have been carefully resolved. The convergence characteristics of a particular model using a given numerical technique are also an important, but most often overlooked, aspect of turbulence model predictions. It is found that some convergence characteristics could be sacrificed for more accurate near-wall prediction. However, even this gain in accuracy is not sufficient to model the effects of an external pressure gradient imposed by a shock-wave/ boundary-layer interaction. Additional work on turbulence models, especially for compressibility, is required since the solutions obtained with base line turbulence are in only reasonable agreement with the experimental data for the viscous interaction problems.

\section{SUBJECT TERMS}

Turbulence model; Supersonic flows; Implicit technique; Shock wave-boundary layer interactions; $\mathrm{k}-\varepsilon$ model

15. NUMBER OF PAGES

17. SECURITY CLASSIFICATION OF REPORT

Unclassified

18. SECURITY CLASSIFICATION
OF THIS PAGE
Unclassified

Unclassified
19. SECURITY CLASSIFICATION OF ABSTRACT Unclassified 